\title{
Reset Control System - View of Internal Concept
}

\author{
Hussam Mohammed-ElHassan Ibrahim Dafaalla ${ }^{1}$, Dalia Mahmoud ${ }^{2}$ \\ ${ }^{1}$ Alneelain University, Faculty of Engineering, Control Systems Engineering, Khartoum, Sudan \\ ${ }^{2}$ Alneelain University, Faculty of Engineering, Assistant Professor, Head of Control Systems Department, Khartoum, Sudan
}

\begin{abstract}
Reset controllers has began in 1959 with the Clegg integrator; It was not until the Horowitz work on quantitative design procedure was developed in 1974, firstly around the Clegg integrator in Krishnan and Horowitz and then another progress has made in 1975 around the first order reset element (FORE) in Horowitz and Rosenbaum.
\end{abstract}

Keywords: Clegg Integrator; Base Linear System; Reset Control

\section{Introduction}

The mechanism of a reset controller is a strategy that resets the controller to its' Zero state or part of it, when a certain condition happen. This condition which triggers the reset action can be explained with an example of plant is a standard linear time invariant (LTI) system with a transfer function $P(s)$ as in Fig. 1.1 below. The broken box of $C(s)$ indicates to its' operation as a Reset Controller.

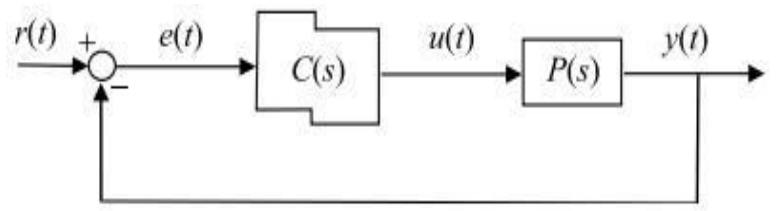

Suppose that the linear system $C(s)$ is given by a first order transfer function $\rightarrow C(s)=\frac{K}{s+a^{\prime}}$

Thus the base linear dynamics would be $\dot{u}=-a u+K e$. Since our assumption for reset condition is Zero crossing, then the input $(e=0)$ and that the reset action is the zero reset of the controller state.

The equations which represent this reset controller as a First Order Reset Element ( FORE)

$$
\begin{cases}u(t)=-a u(t)+K e(t) & \text { when } e(t) \neq 0 \\ u(t+)=0 & \text { when } e(t)=0 .\end{cases}
$$

The first equation called flow mode and it represents the continuous dynamics behavior of the process. The second equation called jump mode and it represents the impulsive dynamics of the process.

Let's compare the behavior of the plant when values of $\mathrm{K}=\mathrm{a}$ $=1$ for the controller and $P(s)=(\mathrm{s}+1) /(\mathrm{s}(\mathrm{s}+0.2))$.

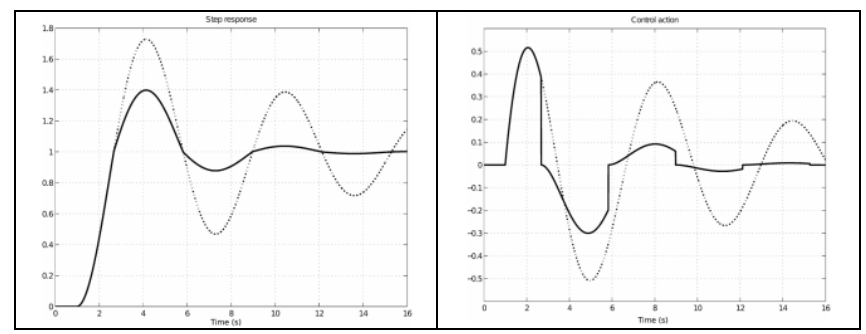

\begin{tabular}{|c|c|}
\hline $\begin{array}{c}\text { Fig. 1.2. Step response } y(t) \text { of } \\
\text { the reset system is "solid" and } \\
\text { base linear system is "dotted". }\end{array}$ & $\begin{array}{c}\text { Fig. 1.3. Control action } u(t) \text { of } \\
\text { the reset system is "solid" and } \\
\text { base linear system is "dotted". }\end{array}$ \\
\hline $\begin{array}{c}\text { The base linear system is very } \\
\text { underdamped, with a large } \\
\text { overshoot }>70 \% \text {. The reset } \\
\text { system response "dotted" line } \\
\text { is much more damped, the } \\
\text { main overshoot decreases to } \\
\text { myster for the reset } \\
\text { system than for the linear } \\
\text { system. Fig. 1.3. Illustrates } \\
\text { clearly the discontinuities of } \\
\text { the reset system from } y\left(t_{k^{-}}\right) \text {to } \\
\text { oscillations disappear in a } \\
\text { quick manner. }\end{array}$ & $y\left(t_{k}+\right)=0$, where $\rightarrow t_{k}$ are the \\
reset times.
\end{tabular}

Conclusion reached there exist some classes of linear plants $P(s)$ that are very difficult to control with linear controllers and there for it's linearly unsolvable trade-offs between competing design objectives.

In control practice, reset control may perform better, but it may also perform worse than a well designed linear compensator, and generally Reset Control should be used carefully.

\section{Clegg integrators and FOREs}

Are very simple, low-order, particular cases of reset controllers. A general reset controller with linear base dynamics would be described by the following equations:

$$
\left\{\begin{array}{l}
\text { (1) } \dot{\mathbf{x}}(t)=\mathrm{Ax}(t)+\mathrm{Be}(t) \rightarrow \text { when } e(t) \neq 0 \\
\text { (2) } \mathbf{x}(t+)=\mathbf{A p x}(t) \rightarrow \text { when } e(t)=0 \\
\text { (3) } \mathbf{u}(t)=\mathbf{C x}(t)+\mathrm{De}(t) \\
\text { (4) } \mathbf{x}(0)=\mathbf{x} 0
\end{array}\right.
$$

If we take the transfer function $(s)=C(s l-A)^{-1} B+D$ then the base linear system of the given is defined by the $1^{\text {st }}$ and $3^{\text {rd }}$ equation, describing the flow mode and holding almost all the time, when $e(t) \neq 0$. The impulsive dynamics, or the jump mode, is triggered by $e(t)=0$. If some of the states are affected by reset and some are not, we can, without loss of generality, re-order states, for example, bring nonreset states firstly and reset states lastly. In this manner, the reset matrix $\mathbf{A} p$ puts in the form of $\mathbf{A} p \operatorname{diag}(1, \ldots, 1, \ldots, 0)$ where $\rightarrow(1, \ldots, 1)=n 1$ and $(0, \ldots, 0)=n 2$

Since, $n 1+n 2=n$. If $n 2=0$, we do have a linear controller. If $0<n 2<n$, we do have what so-called partial reset controller and here only one part of the states are reset states 


\section{International Journal of Science and Research (IJSR) \\ ISSN (Online): 2319-7064}

Index Copernicus Value (2013): 6.14 | Impact Factor (2014): 5.611

but, if $n 2=n$, full reset state would be applied and $\boldsymbol{x}\left(t_{k}+\right)=0$ $\epsilon \boldsymbol{R}^{n}$.

The First Order Reset Element "FORE" in Fig. 1.5 below is showing a particular case of a full reset system with scalar parameters $\left(A, B, C, D, A_{P}\right)=(-a K, 1,0,0)$.

Conclusion of this part, a reset system or a reset controller, is a dynamical system "typically linear and finite-dimensional" endowed with a reset mechanism. We have to answer two questions in-order to design a coherent reset mechanism:

- When should we apply a Reset condition? This amounts to defining the reset condition input $c(t)$ in Fig. 1.4. below so that $c\left(t_{k}\right)=0$ defines the reset instants $t_{k}(k=1,2, \ldots)$. Usually, it is made equal to the tracking error of the control system $c(t)=e(t)$, but other modifications are possible. For example, combinations of $\dot{e}(t)$ and $e(t)$, thresholds for $e(t)$.

- How to apply Reset action? This amounts to defining the after-reset value signal $a(t)$ in Fig. 1.4 so that $u\left(t_{k}+\right)=a(t)$. Normally, reset is applied as zero reset, $a(t)=0$, but, often it is more useful to apply a percentage of reset, $a(t)=p u(t)$ so that $u\left(t_{k}+\right)=p\left(t_{k}\right) u\left(t_{k}\right)$ with $(1>p(t)>0)$.

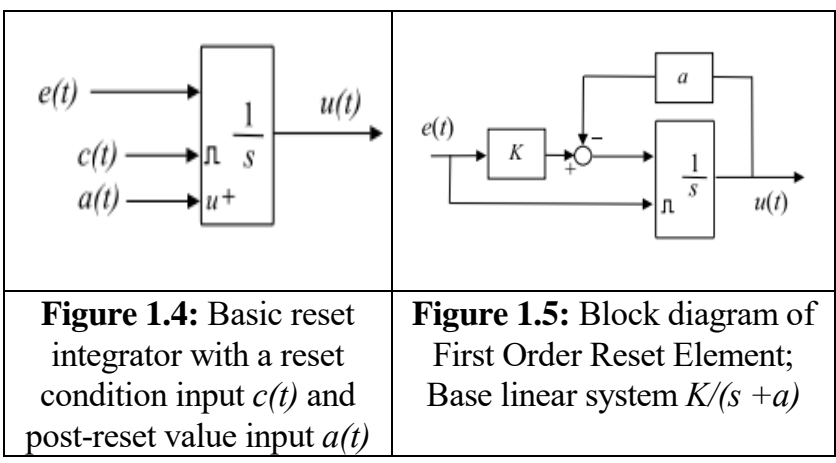

\section{Why Do We Need Reset Control?}

The main reason for using reset controllers is that the idea of just by including mechanism of resetting, we would have the capability of overcoming the fundamental limitations in linear systems. Historically in very beginning this idea was presented by Clegg's integrator as a solution method to improve feedback performance, due to its ability to provide the same modulus attenuation as a linear integrator $(-20$ $\mathrm{dB} / \mathrm{dec})$ but with a phase $\left(\approx-38^{\circ}\right)$ much more favorable in terms of phase margins and robustness. Overcoming linear limitations ability was also implicit in the subsequent proposals of reset control; from the First Order Reset Element (FORE) by Horowitz and his coworkers to the many of recent researches and studies of reset controllers.

\subsection{Reset Control with the Clegg Integrator}

Clegg integrator was proposed for the first time as a complete and quantitative control design procedure by Krishnan and Horowitz. Fig.1.6. below shows a simplified control system diagram, where we can see a two-degree-offreedom structure with pre-filter $F(s)$ acting on the reference $r(t)$, output disturbance $\rightarrow d(t)$, and measurement noise $\rightarrow$ $n(t)$. The plant linear factors may also be given from the controller by $P(s)$, and $G$ is the controller that could be linear or nonlinear.
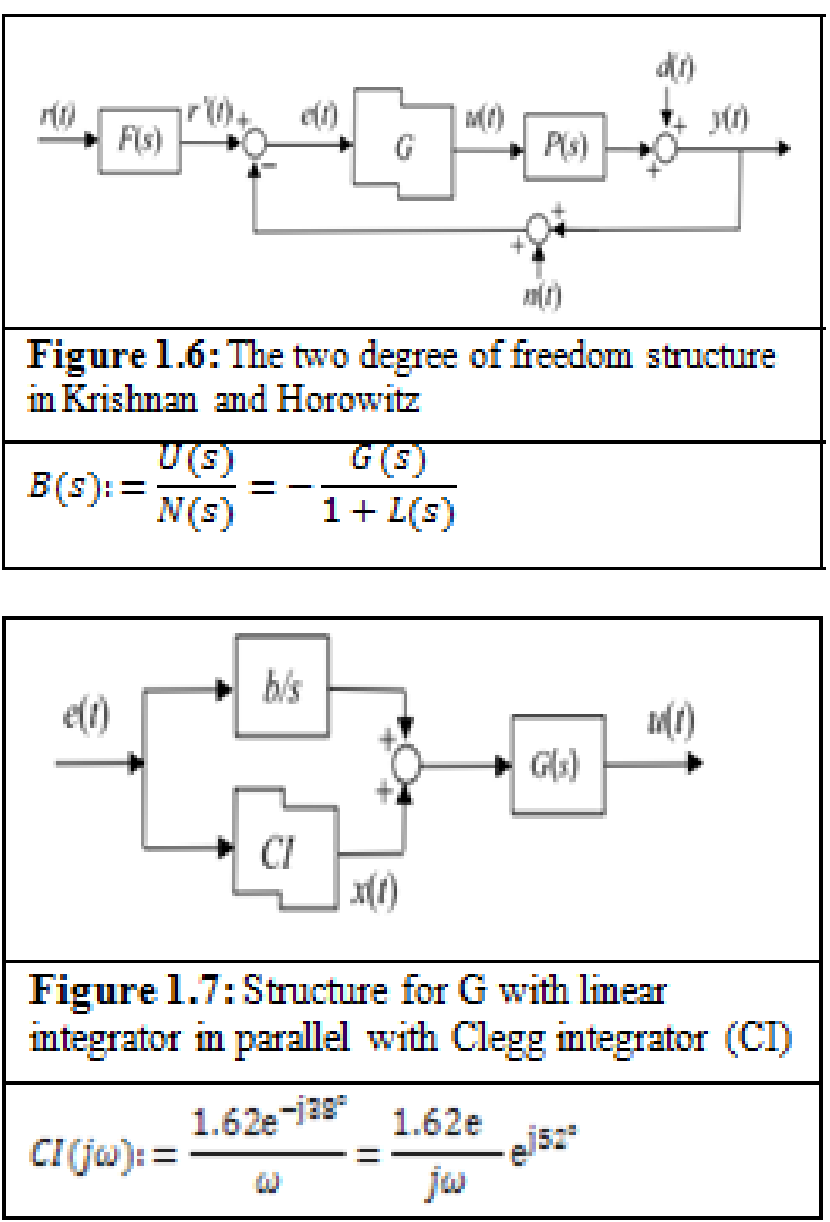

\section{Conclusion}

On this paper, reset control systems with Clegg integrators and FOREs overview. Some backgrounds have been highlighted about the relationship between Clegg integrators and FOREs Reset Control Systems. Due to space limits, it doesn't give the specific examples of the plots of reset control figures, hoping that readers can grasp the concept that help them build solid ground relied upon to look in depth of Reset Control System.

\section{References}

[1] Aangenent, W.H.T.M., Witvoet, G., Heemels, W.P.M.H., van de Molengraft, M.J.G., Stein-buch, M.: Performance analysis of reset control systems. Int. J. Robust Nonlinear Control 20(11), (2009).

[2] Åström, K.J.: Limitations on control system performance. Eur. J. Control 6, (2000).

[3] Barreiro, A., Baflos, A.: Delay-dependent stability of reset systems. Automatica (2010).

[4] Beker, O., Hollot, C.V., Chait, Y, Han, H.: Plant with integrator: an example of reset control overcoming limitations of linear systems. IEEE Trans. Autom. Control (2001).

[5] Carrasco, J., Baflos, A., van der Schaft, A.: A passivity approach to reset control of nonlinear systems. In: 34th 
Annual Conference of the IEEE Industrial Electronics Society, Orlando, EE.UU (2008).

[6] Horowitz, I.M., Rosenbaum, P.: Nonlinear design for cost of feedback reduction in systems with large parameter uncertainty. Int. J. Control (1975).

[7] Chait, Y., Hollot, C.V.: On Horowitz's contributions to reset control. Int. J. Robust Nonlinear Control 12, 335355 (2002).

\section{Author Profile}

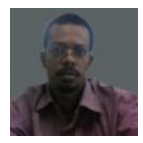

Hussam Mohammed-ElHassan Ibrahim Dafaalla received his B.Sc. Engineering from European University of Lefke, Turkish Higher Education Board YÖK. He has over 5 years experience in Telecommunication industry at Saudi Arabia. Now he is doing his supplementary research on Master Degree at Sudan.

Dalia Mahmoud Assistant Professor and currently Head of Control Systems Department at Alneelain University -Faculty of Engineering in Sudan. Dalia holds a PhD degree and she has more than 10 years Experience in higher Education field. 